\title{
Evidence for Two Functionally Distinct Subpopulations of Neurons Within the Rat Striatum
}

\author{
Eric S. Nisenbaum, William B. Orr, and Theodore W. Berger \\ Departments of Behavioral Neuroscience and Psychiatry, Center for Neuroscience, University of Pittsburgh, Pittsburgh, \\ Pennsylvania 15260
}

\begin{abstract}
Type I and Type II extracellular action potential waveforms were recorded from the rat striatum and studied with respect to their dependence on recording conditions, response to paired impulse stimulation of the corticostriatal pathway, and iontophoretic application of dopamine (DA). Results showed that the distinguishing characteristics of Type I and Type II waveforms are relatively independent of the degree of filtering, distance of the electrode tip from the target neuron, type of recording electrode, and firing rate of the neuron. Very low impedance electrodes, however, were found to mask the difference in spike shape. Electrical stimulation of cortical afferents results in excitation of both action potential waveforms, though the Type II class exhibits a significantly shorter latency than the Type I class. Paired impulse analyses revealed that both waveforms exhibit variation in the probability of discharge (facilitation or inhibition) to the second impulse of each impulse pair that are a function of the interimpulse interval. Most importantly, however, the probabilities of discharge of Type I and Type II neurons to the second impulse are inversely related, i.e., when one cell type exhibits facilitation, the other displays inhibition. These data demonstrate that Type I and Type II waveforms represent the activity of functionally different subpopulations of striatal neurons. Moreover, Type II neurons are found much more often than Type I cells, suggesting that the 2 cell classes may be represented with different frequencies within striatum. Finally, Type II neurons display at least a 5 times greater sensitivity to iontophoretically applied DA than Type I cells, suggesting that the 2 cell populations also are affected differentially by dopaminergic input from the substantia nigra.
\end{abstract}

Cellular analyses using morphological, biochemical, and immunocytochemical methods have shown the striatum to be a heterogeneous structure in terms of its anatomy (Kemp and Powell, 1971; Pasik et al., 1979; Chang et al., 1982) and neurotransmitter content (for review, see Graybiel and Ragsdale, 1983). Consistent with this characterization, electrophysiological studies have found that striatal neurons exhibit a variety of responses to stimulation of extrinsic afferents (McLennan and

Received Aug. 19, 1987; revised Feb. 29, 1988; accepted Mar. 14, 1988.

This research was supported by grants from NINCDS (NS19608) and NIMH (MH00343). We are grateful for suggestions made by Drs. Anthony A. Grace, Fdward M. Stricker, and Michael J. Zigmond in the preparation of this manuscript. E.S.N. was supported by a training grant from NIMH (MH18273).

Correspondence should be addressed to Theodore $W$. Berger, 465 Crawford Hall, Department of Behavioral Neuroscience, University of Pittsburgh, Pittsburgh, PA 15260.

Copyright (C) 1988 Society for Neuroscience $0270-6474 / 88 / 114138-13 \$ 02.00 / 0$
York, 1967; Connor, 1970; Feltz and Albe-Fessard, 1972; Gonzalez-Vegas, 1974; Kitai et al., 1975), iontophoretic application of putative neurotransmitters (Bloom et al., 1965; Feltz and DeChamplain, 1972; Bunney and Aghajanian, 1973; Bevan et al., 1975; Kitai et al., 1976; Chiodo and Berger, 1986), and a considerable range of spontaneous discharge patterns (Wilson and Groves, 1981). Because differences in these parameters have not been found to correlate, however, it has not been possible to conclusively identify subclasses of striatal neurons on the basis of their functional properties.

Skirboll and Bunney (1979) first reported that 2 different types of action potential waveforms can be recorded extracellularly from the rat striatum: Type I waveforms have an initial negativity and are smaller in amplitude than Type II waveforms, which have an initial positivity. They also found that the 2 waveforms respond differently to both acute and chronic haloperidol treatment, suggesting that they reflect functionally different subpopulations of neurons. However, a single neuron can exhibit different extracellular amplitudes and action potential waveforms depending on the distance and orientation of the cell relative to the recording electrode, and on the electrical characteristics of the electrode (e.g., impedance, shape, and material). Moreover, because the same cell could not be recorded from both before and after drug treatment, the possibility cannot be eliminated that the haloperidol-induced alterations in firing rate led to changes in the extracellular waveform. Thus, the differences in waveform and pharmacological response, although suggestive of separate striatal cell populations, are not sufficient to establish their identity.

In the present study, we have conducted a detailed examination of Type I and Type II extracellular waveforms to determine if they are only products of particular recording conditions or level of cell activity. In addition, we have investigated the hypothesis that the 2 waveforms represent functionally distinct action potential generators by using paired impulse stimulation of the corticostriatal pathway to examine potential differences in their evoked response properties. Finally, we have determined the sensitivity of the 2 waveforms to iontophoretic application of dopamine (DA). Here we report that Type I and Type II action potential waveforms are independent of most recording conditions (e.g., single versus multibarrel electrodes) and firing rate of the cell. The identity of both waveforms also is independent of distance of the electrode from the recorded neuron over a fairly wide range, though when recording distance was great (i.e., when spike amplitude is small) their distinction becomes difficult. Very low impedance electrodes, however, were found to mask the difference in spike shape. Our central findings are that the 2 classes of waveforms exhibit qualitatively different 
responses to paired impulse stimulation of their cortical afferents, demonstrating that Type I and Type II waveforms represent 2 functionally different cell populations of striatal neurons. In addition, Type I and Type II neurons exhibit significantly different sensitivities to DA, suggesting that the 2 cell classes are likely to be affected differentially by dopaminergic input from the substantia nigra.

\section{Materials and Methods}

All animals were male, Sprague-Dawley albino rats $(n=52)$ weighing $250-450 \mathrm{gm}$ at the time of surgery. In preparation for electrophysiological recording, animals were anesthetized with chloral hydrate (40 $\mathrm{mg} / \mathrm{kg}$, i.p.), and a catheter was inserted into the femoral vein for administration of supplemental doses of anesthesia. The level of anesthesia was monitored periodically by examining corneal reflexes in response to contact of the cornea with a moist cotton swab and muscle flexion or change in respiration rate caused by an ear pinch applied with forceps. The depth of anesthesia was maintained such that little, if any, response occurred to these stimuli.

The extracellular responses of single acton potential waveforms were recorded using either 1- or 5-barrel glass micropipettes. Single-barrel pipettes were filled with $2 \mathrm{M} \mathrm{NaCl}$ saturated with Pontamine Sky Blue and had input impedances of $1-10 \mathrm{M} \Omega$ when tested in vitro at $350 \mathrm{~Hz}$. When 5-barrel pipettes were used, recordings were made through the center barrels, which also were filled with $2 \mathrm{~m} \mathrm{NaCl}$ saturated with Pontamine Sky Blue and had input impedances of 5-18 M 2 . All extracellular action potentials $(n=354)$ were recorded from the anterodorsal portion of the striatum and were isolated by their orthodromic response to single stimulation impulses ( $0.1 \mathrm{msec}$ duration, biphasic) applied to the corticostriatal pathway. More specifically, bipolar stimulating electrodes were placed $2.0 \mathrm{~mm}$ anterior to bregma, $2.5 \mathrm{~mm}$ ventral to the cortical surface, and either 1.75 or $3.65 \mathrm{~mm}$ lateral to the midline for activating neurons in either medial or lateral aspects of the striatum, respectively. Action potential discharges of single neurons were recorded from a striatal region bounded by planes $1.0 \mathrm{~mm}$ anterior to bregma, from $1.70-3.60 \mathrm{~mm}$ lateral to the midline, and $2.8-6.0 \mathrm{~mm}$ ventral to the cortical surface.

All recorded action potentials were classified as either Type I or Type II according to waveform criteria established by Skirboll and Bunney (1979). That is, action potentials were classified as Type I $(n=48)$ if they appeared as negative-positive or as Type II $(n=306)$ if they appeared as positive-negative (Fig. 1). Even the presence of a relatively small amplitude initial positivity (see Results) led to classification as a Type II waveform.

In some experiments, the number of Type I and Type II waveforms was counted during movement of the recording electrode in the dorsoventral plane to obtain an estimate of the frequency with which these waveforms were encountered. For these experiments, action potentials were recorded from 4 mediolateral locations $(1.70,1.75,3.60$, and 3.65 $\mathrm{mm}$ lateral to the midline) and at either 0.5 or $1.5 \mathrm{~mm}$ anterior to bregma. Only those neurons encountered within $2.8-6.0 \mathrm{~mm}$ ventral to the cortical surface were counted. As described above, all neurons were localized by their orthodromic response to stimulation of the corticostriatal pathway, and the number of each type recorded per electrode track was counted using both high (3.5-5.5 $\mathrm{M} \Omega$ ) and low (1.0-2.0 M $\Omega$ ) impedance electrodes.

Additional experiments investigated the extent to which differences in the shapes of Type I and Type II waveforms depend on the degree of capacitive coupling used in filtering the signals after amplification. For this determination, photographs were taken of action potential waveshapes evoked by orthodromic stimulation, and the degree of highpass filtering was varied for each waveform. Limits for high-pass filtering ( $28 \mathrm{~dB} /$ octave) were $1,10,100,500$, and $1000 \mathrm{~Hz}$; low-pass filtering was held constant at $5 \mathrm{kHz}$.

Paired impulse stimulation. After isolation and classification, further stimulation of the corticostriatal pathway was applied using a paired impulse format. Stimulus intensity was adjusted so that the probability of discharge to the first impulse of each pair was either approximately 0.50 (threshold) or $>0.80$ (suprathreshold). The probability of eliciting a response to the second impulse of each pair then was examined as a function of interstimulus interval. For 55 of 325 neurons, paired impulse stimulation was conducted by computer control and involved the use of a large number of interimpulse intervals; for the remaining neurons,
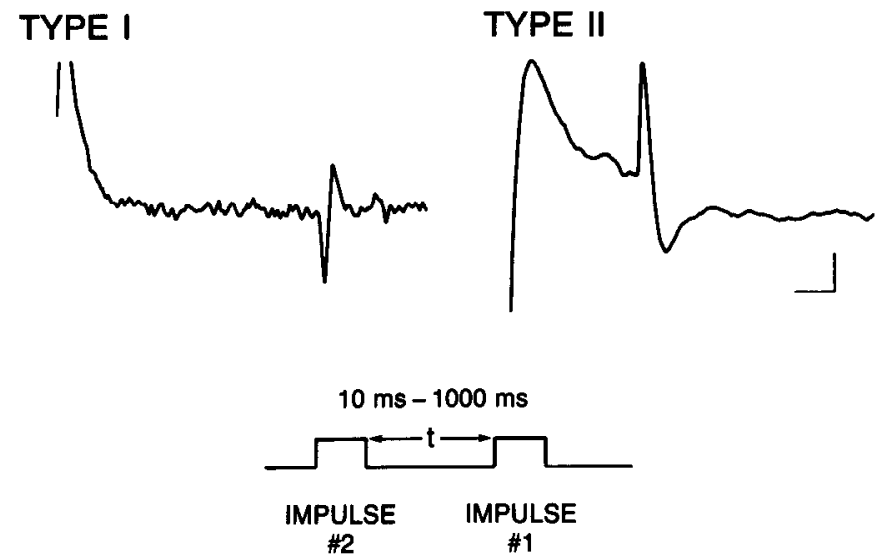

Figure 1. Extracellular responses of orthodromically activated striatal Type I and Type II action potential waveforms to stimulation of the corticostriatal pathway. Calibration bars: Type I, $200 \mu \mathrm{V}$ and $2 \mathrm{msec}$; Type II, $100 \mu \mathrm{V}$ and $2 \mathrm{msec}$. The high-pass filtering cut-off was 300 $\mathrm{Hz}$; low-pass filtering cut-off was $5 \mathrm{kHz}$ (Type I) and $10 \mathrm{kHz}$ (Type II). Positive is up in this and all similar figures.

a smaller, select number of intervals were tested manually. For the cases tested by computer, a pair of impulses was delivered once every 5-10 sec, alternating between short and long interstimulus intervals. The intervals tested were (1) every $10 \mathrm{msec}$ from 10 to $200 \mathrm{msec}$, (2) every $20 \mathrm{msec}$ from 200 to $500 \mathrm{msec}$, and (3) every $50 \mathrm{msec}$ from 500-1000 msec. At least 4 and as many as 16 repetitions of each paired impulse interval were completed for each cell, and all intervals were given once before repetition began.

In response to each stimulation impulse, Type I and Type II waveforms were amplified and bandpass-filtered using a low-frequency cutoff between $300-1 \mathrm{kHz}$ and a high-frequency cut-off of $5 \mathrm{kHz}$. The filtered responses were digitized continuously by a computer and stored. Data were analyzed off-line using software algorithms that detected evoked action potential discharges (verified visually). The following was then calculated for each cell: (1) the probability of discharge in response to the first impulse of all pairs of impulses, which was defined as the baseline response, and (2) the probabilities of discharge to the second impulse of each interstimulus interval, which were averaged and normalized relative to the baseline value. Because 1-2 hr could be required to complete testing of a given cell, only 1-4 neurons were recorded from each animal when the entire range of interstimulus intervals was used.

Iontophoretic application of DA. Sensitivity to DA was tested using iontophoretic procedures and 5-barrel micropipettes. Extracellular action potentials were recorded through the center barrel (see above). Of the 4 outcr barrcls, one was filled with L-glutamic acid (monosodium salt, $0.1 \mathrm{M}, \mathrm{pH} 8.0$ ), another was filled with dopamine hydrochloride (DA, $0.1 \mathrm{M}, \mathrm{pH} 4.0$ ), and a third was filled with the DA receptor antagonist trifluoperazine dihydrochloride $(0.01 \mathrm{M}$ in $0.01 \mathrm{M}$ tartaric acid, $\mathrm{pH} 4.0$ ). When a drug was not being ejected, a 10-15 nA retention current was applied to the barrel. The fourth barrel contained $4 \mathrm{M} \mathrm{NaCl}$, which was used for automatic neutralization of tip currents.

A window discriminator was used to convert Type $\mathrm{I}(n=15)$ and Type II $(n=14)$ waveforms into TTL-level digital pulses. The digital pulses then were integrated on-line by computer, which also calculated the average firing rate and percentage change in firing rate induced by iontophoretic drug application. Integration of the output pulses was displayed on a chart recorder during the course of each experiment.

Because both Type I and Type II action potentials have low, nearzero spontaneous discharge rates, iontophoretic application of glutamate was used to increase their level of baseline firing. A dose-response relation for the effect of iontophoretically applied DA on glutamateevoked activity then was measured. To control for possible differences in the effects of DA due to differences in absolute firing rate of the target neuron, the amount of glutamate ejected was adjusted to obtain discharge rates within the same range for both types of waveforms. The mean number of spikes per second was $3.9 \pm 1.5$ (mean \pm SD) for Type I waveforms and $3.3 \pm 2.0$ for Type II waveforms, rates that are not statistically different $[t(25)=0.91]$. The ranges of glutamate-induced firing rates for the 2 classes of action potential events also were very 
A
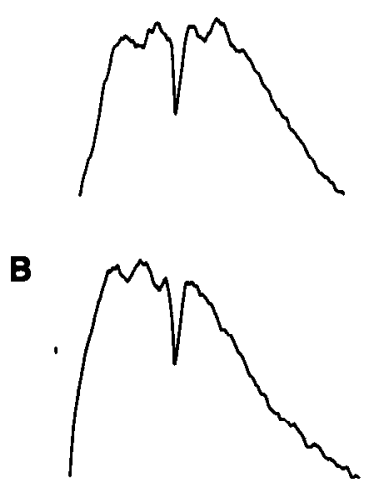

C

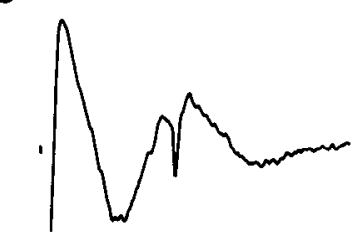

D

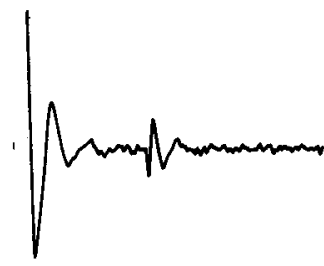

E

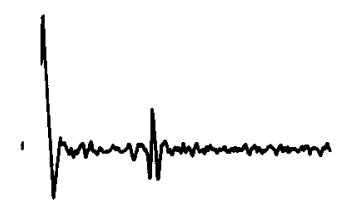

$\mathbf{F}$

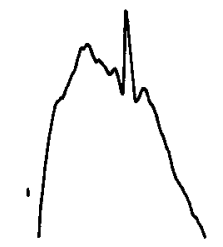

G

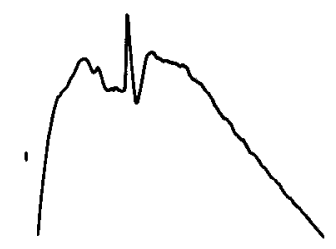

H

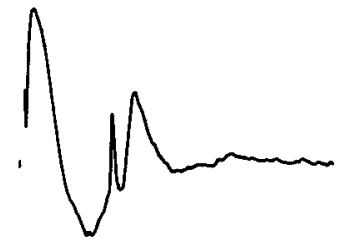

I

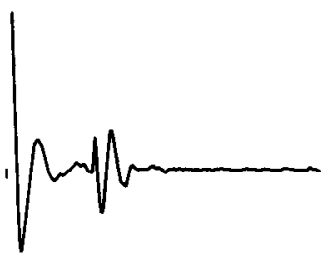

$\mathbf{J}$

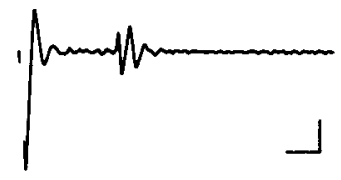

Figure 2. Effects of varying the high-pass filtering cut-off on the shapes of Type I $(A-E)$ an Type II $(F-J)$ waveforms. The high-pass filtering cut-off was increased in the following increments: $1 \mathrm{~Hz}(A$ and $F), 10$ $\mathrm{Hz}(B$ and $G), 100 \mathrm{~Hz}(C$ and $H), 500 \mathrm{~Hz}(D$ and $I)$, and $1000 \mathrm{~Hz}(E$ and $J$. Low-pass filtering was held constant at $5 \mathrm{kHz}$. Note that although the degree of filtering affected the shapes of Type I and Type II waveforms, the distinguishing characteristics of Type I (initial negativity) and Type II (initial positivity) waveforms were unaltered. Calibration bars, $100 \mu \mathrm{V}$ and $2 \mathrm{msec}$ except $E$, in which bars represent $50 \mu \mathrm{V}$ and 2 msec.

similar (Type I waveforms, 1.6-6.2 spikes/sec; Type II waveforms, 1.1$6.2 \mathrm{spikes} / \mathrm{sec}$ ).

The low glutamate-evoked activity of most neurons proved quite variable, so that it was difficult to maintain steady baseline firing. For this reason, DA was applied for $3.5 \mathrm{~min}$ at each level of ejection current for DA to obtain a sufficient sample of spike discharges for accurate measurement. A minimum of 4 min was used to establish the baseline rate of each cell. For most neurons (Type I, $n=12$; Type II, $n=9$ ), DA was applied continuously, first with an ejection current of $5 \mathrm{nA}$ and then with increasing currents of $10,20,30,40,50,60$, and $70 \mathrm{nA}$ (maximum) or until ncuronal activity was inhibited completely. After it became clear that Type II neurons display a much greater sensitivity to DA, however, this cell type also was tested using $15 \mathrm{nA}$. The first 30 sec of each drug application was not included in the calculations of DAinduced percentage change in firing rate. After DA application was terminated, glutamate-induced firing was allowed to recover to baseline levels; only cells exhibiting recovery were included in subsequent analyses. In some cases, after recovery, iontophoresis of DA was repeated

with simultaneous application of trifluoperazine (TFP, 5-10 nA; $n=$ 10) to test for the specificity of DA-induced changes in cell firing.

For some neurons, the effects of a noncontinuous procedure for applying DA also were examined. For neurons with a Type I waveform $(n=3)$ DA was applied at $10,20,30,40,60$, or $70 \mathrm{nA}$ for $3.5 \mathrm{~min}$ intervals, with interdrug intervals of $3.5 \mathrm{~min}$; for neurons with a Type II waveform $(n-5)$, DA was applied with ejection currents of 5,10 , or $20 \mathrm{nA}$. The order of ejection currents was selected such that a high current (40 or $60 \mathrm{nA}$ for Type I cells, and 10 or $20 \mathrm{nA}$ for Type II cells) was repeated before each lower current to control for warm-up effects. In a few cases $(n=4)$, responses to both continuously and noncontinuously applied DA were obtained for comparison.

\section{Results}

\section{Effects of filtering on Type I and Type II waveforms}

The characteristic shapes of Type I and Type II waveforms were found to be relatively independent of distortions produced by the degree of capacitive coupling due to filtering. For example, varying the high-pass filtering cut-off from $1 \mathrm{~Hz}$ to $1 \mathrm{kHz}$ did not change the basic waveform properties (Fig. 2); Type I waveforms always displayed an initial negative-going potential, and Type II waveforms always displayed an initial positive-going potential. Increased filtering did have the effect of changing the Type I waveform from a monophasic to a biphasic form and of reducing the amplitude of its negative component, though the fundamental characteristic of an initial negativity was not altered (Fig. 2A-E). The degree of high-pass filtering was found to be inversely related to the amplitude of the initial positivity of Type II waveforms. Thus, as high-pass filtering was increased progressively from $1 \mathrm{~Hz}$ to $1 \mathrm{kHz}$ the amplitude of the initial positivity of a Type II waveform was diminished (Fig. $2 F-J$ ), and its distinction from the Type I class became more difficult.

\section{Effects of recording distance on Type I and Type II waveforms}

Type I and Type II action potential waveforms exhibited amplitudes of different sizes that presumably were due, at least in part, to differences in the distances between the electrode tip and the recorded neurons (Fig. 3). Although we did not investigate the effect of this variable in a quantitative manner, the incidence of Type I or Type II waveforms was not observed to correlate with spike amplitude and, thus, appeared to be independent of recording distance. In addition, the characteristic shape of neither Type I nor Type II action potentials was altered during "drift" of the electrode during the course of a recording. However, when Type II waveforms were associated with spikes of relatively small amplitude (e.g., $50 \mu \mathrm{V}$ ), such as when a cell was first encountered, the initial positivity was reduced and the spike was difficult to distinguish from a Type I waveform. As the recording electrode was advanced further toward the cell, the initial positivity became more prominent in conjunction with increasing amplitude (Fig. $3 F-J$ ). The apparent lack of dependence of waveform on recording distance was tested explicitly by moving the electrode during the course of many recordings. Displacement of the recording tip by as much as 100 $\mu \mathrm{m}$ resulted only in changes of amplitude and not shape of the recorded waveform. Although movement of the electrode over such a distance undoubtedly involved movement of the cell as well through friction of the electrode with the surrounding tissue, the results of such tests demonstrate that the fundamental characteristics of the 2 waveforms are not critically dependent on distance of the recording electrode over a fairly wide range. 
A

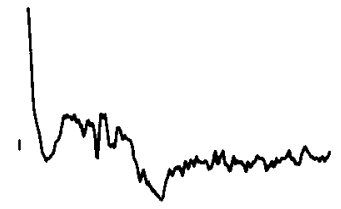

B

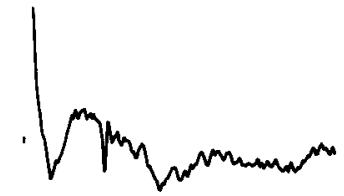

C

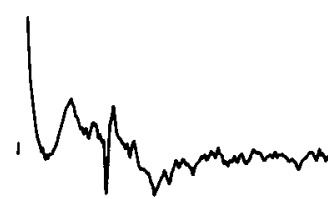

D

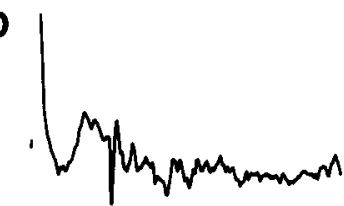

E

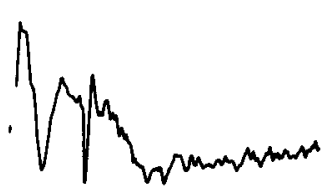

F

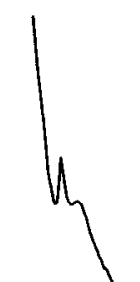

G

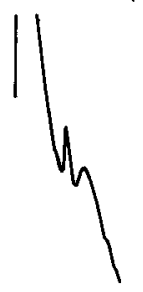

H

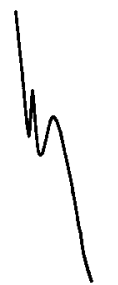

I

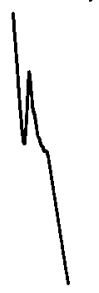

J

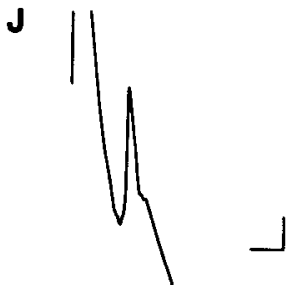

Figure 3. Effects of varying the distance of the recording electrode on the shapes of Type I $(A-E)$ and Type II $(F-J)$ waveforms. When the recording electrode was moved approximately $100 \mu \mathrm{m}$ from the point of first locating either a Type I or Type II waveform, only a change in amplitude was observed. Calibration bars: $A-E, 50 \mu \mathrm{V}, 2$ msec; $F-J$, $100 \mu \mathrm{V}, 2$ msec. The high- and low-pass filtering cut-offs were $300 \mathrm{~Hz}$ and $5 \mathrm{kHz}$, respectively.

\section{Effects of electrode impedance on Type I and Type II waveforms}

When electrodes with lower impedances (1-2 M $\Omega$ ) were used, the initial positivity of Type II waveforms was diminished, in much the same way as under conditions of increased high-pass filtering and recordings from relatively large distances (see above). This effect of electrode impedance was studied in a quantitative manner, and the results of that analysis are described below (see Paired impulse stimulation of the corticostriatal pathway).

\section{Initial segment/somatodendritic (IS/SD) action potential components}

The presence of an initial segment/somatodendritic(IS/SD) break was found to be associated frequently with Type II waveforms (Fig. 4). We did not observe an IS/SD break in association with a Type I waveform.
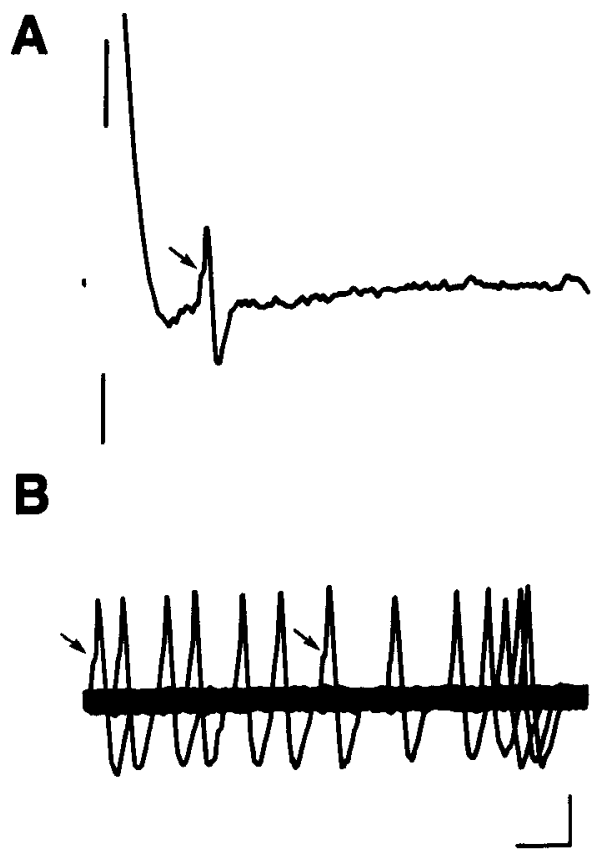

Figure 4. Examples of IS/SD breaks associated with Type II waveforms. Arrows denote the break between the IS and SD action potential components of a Type II waveform in response to a single stimulation impulse applied to the corticostriatal pathway $(A)$ or during spontaneous discharge $(B)$. Note that the IS/SD break is not always obvious. Calibration bars, $50 \mu \mathrm{V}, 2$ msec. The high- and low-pass filtering cut-offs were $300 \mathrm{~Hz}$ and $5 \mathrm{kHz}$, respectively.

\section{Latencies to activation for Type I and Type II waveforms}

Latencies to activation for Type I and Type II waveforms in response to single impulse stimulation of the corticostriatal pathway also were found to be different. At threshold stimulation intensities, Type I waveforms responded with an average latency of $8.7 \mathrm{msec}( \pm 3.8)$, whereas Type II waveforms responded with an average latency of $7.0 \mathrm{msec}( \pm 1.5)$. This difference in mean latencies to spike discharge for the 2 waveforms was statistically significant $(t=2.82, p<0.01)$.

\section{Paired impulse stimulation of the corticostriatal pathway}

Threshold stimulation. Results showed that paired impulse stimulation of the corticostriatal pathway elicited markedly different responses from Type I and Type II waveforms over the entire range of interstimulus intervals tested. When stimulation intensity was near threshold, the probability of a Type I action potential discharge to the second impulse was increased in response to interstimulus intervals of approximately $10-30 \mathrm{msec}$ (Figs. 5A, left panel, and 6A) for all but one of the waveforms recorded. This facilitative response showed an average peak probability of $0.84 \pm 0.04$ (mean \pm SEM). In response to longer interstimulus intervals of approximately 50-240 msec, Type I waveforms displayed a robust inhibition (Fig. 6B). The decrease in probability of firing approached 0.00 at these intervals, with the average peak probability of $0.09 \pm 0.04$. Following this inhibition, a second facilitative response was seen when interstimulus intervals of approximately $270-750 \mathrm{msec}$ were used. Either a return to baseline responding or a decrease in the probability of firing of Type I waveforms was found for interstimulus intervals of approximately $850-1000 \mathrm{msec}$. The magnitude of this second inhibitory response was never as great as that ob- 


\section{A. TYPE I}

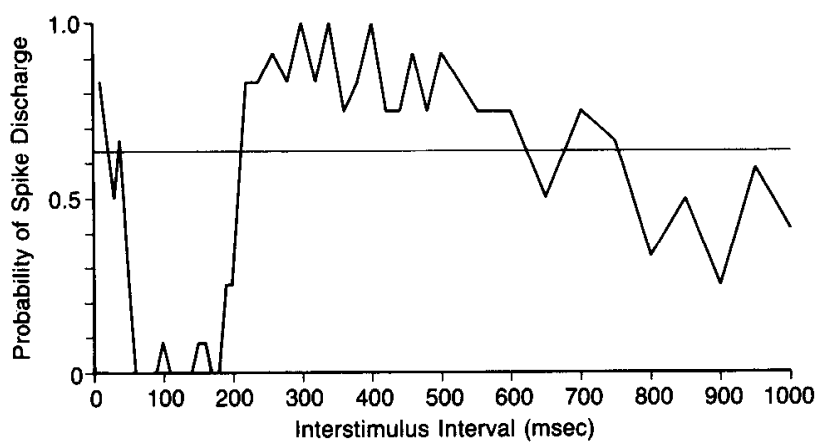

B. TYPE II

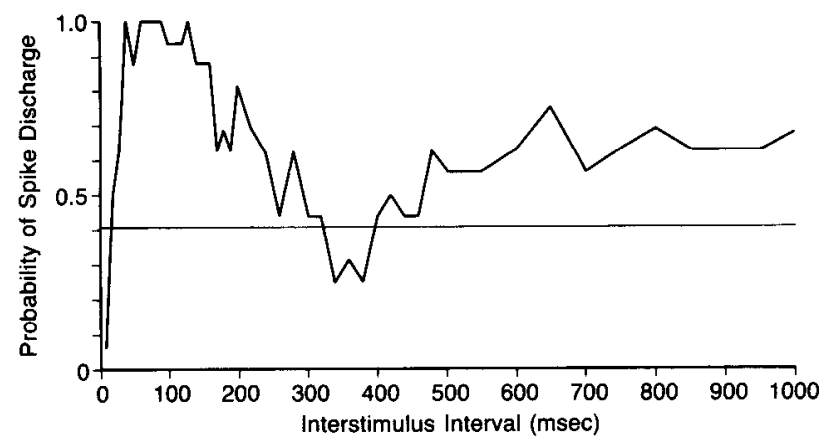

C. TYPE II

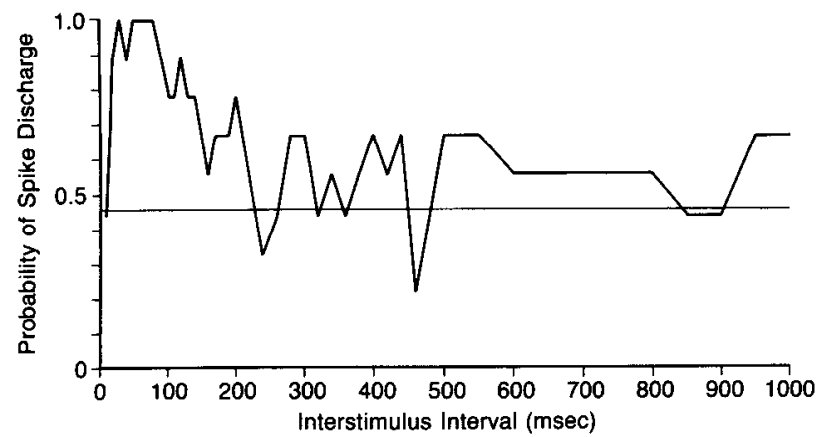

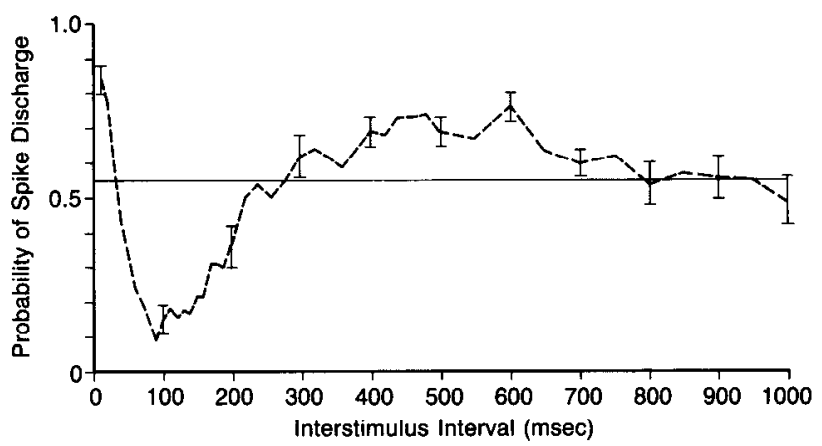
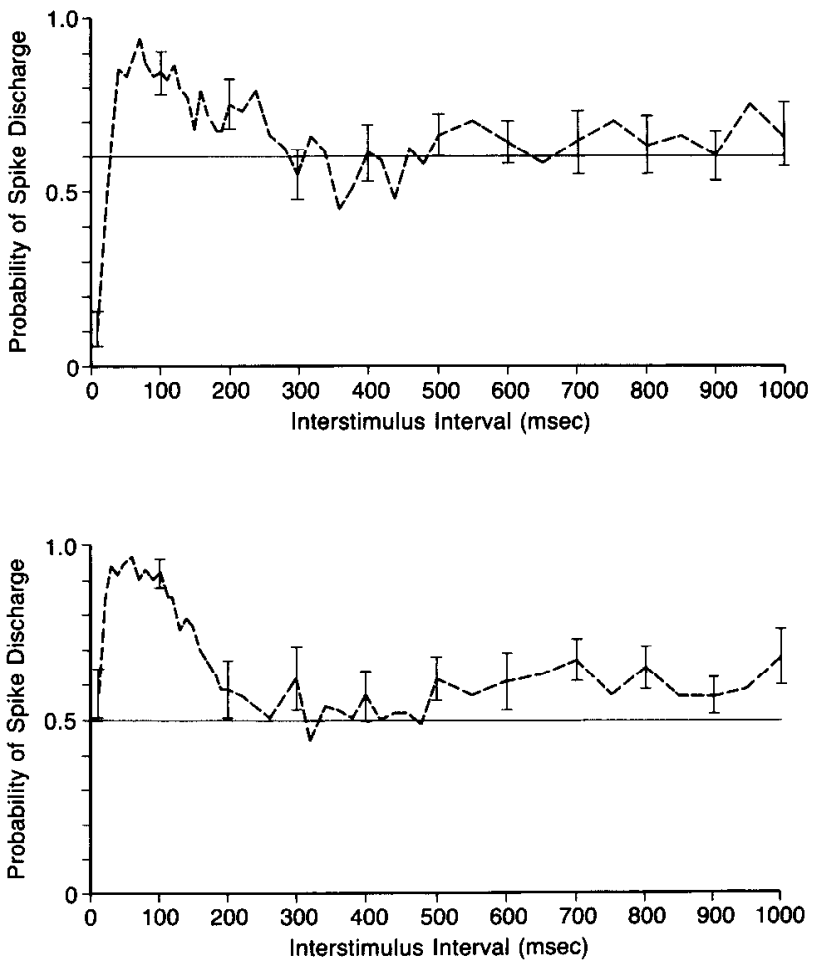

Figure 5. Paired impulse curves for striatal Type I $(A)$ and Type II ( $B$ and $C$ ) waveforms at stimulation intensities approximately equal to threshold. The probabilites of spike discharge to the second impulse of each pair are presented as a function of interstimulus interval. Horizontal lines are equal to the probability of action potential discharge to the first impulse of all pairs of impulses. Left and right panels represent, respectively, single and average paired impulse profiles for Type I waveforms $(A)$, Type II waveforms, exhibiting short-interval inhibition $(B)$, and Type II waveforms not exhibiting short-interval inhibition $(C)$. The bars in the average paired impulse curves (right panels) indicate SEM.

served at shorter intervals. Thus, over the range of interstimulus intervals tested, the paired impulse curves generated by Type I waveforms were characterized by a facilitation-inhibition-facilitation response pattern (Fig. $5 A$, right panel).

Of all the cells tested, only one Type I waveform did not show this pattern. Instead, it displayed no consistent variation in probability of spike discharge to the sccond impulse for any of the interstimulus intervals tested. This neuron also responded to orthodromic activation with a latency to discharge $(16 \mathrm{msec})$ that was much longer than that observed for other Type I waveforms.

Striatal cells exhibiting Type II waveforms displayed a paired impulse profile that was inversely related to that of Type I neurons (Fig. 5B, left panel). For example, when threshold stimulation was used, $50 \%$ of the Type II waveforms showed an initial decrease in the probability of spike discharge in reponse to interstimulus intervals of $10-30 \mathrm{msec}$ (Fig. $7 A$ ). The average peak probability of this inhibitory response was $0.11 \pm 0.13$. Interstimulus intervals between 30 and approximately $250 \mathrm{msec}$ resulted in a facilitation of all Type II waveforms such that the probability of spike discharge approached $1.00(0.94 \pm 0.04$; Fig. 7C). Following this facilitation, and again in contrast to Type I waveforms, Type II waveforms exhibited a decrease in probability of firing for intervals of approximately $280-500 \mathrm{msec}$. Responses of Type II waveforms returned to baseline or showed a second facilitation in response to intervals greater than 600 msec. This second facilitative response never was as great as the initial facilitation.

Thus, the only substantial variation among Type II waveforms when tested using threshold stimulation intensity was the 
A
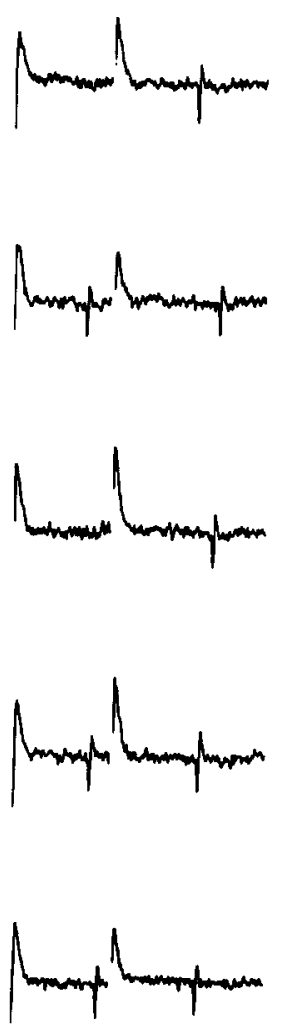

B
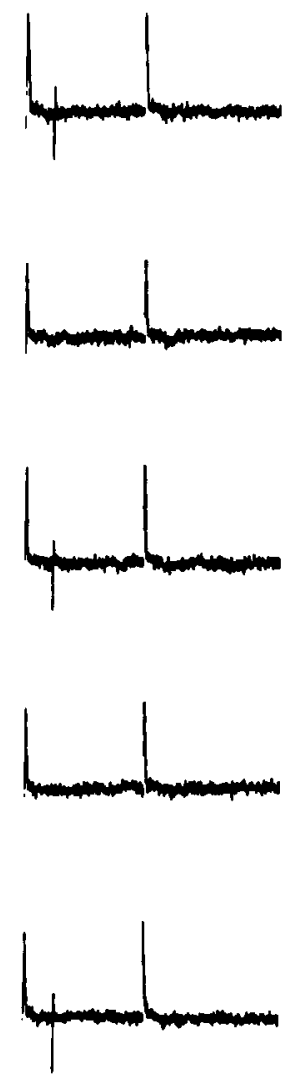

C
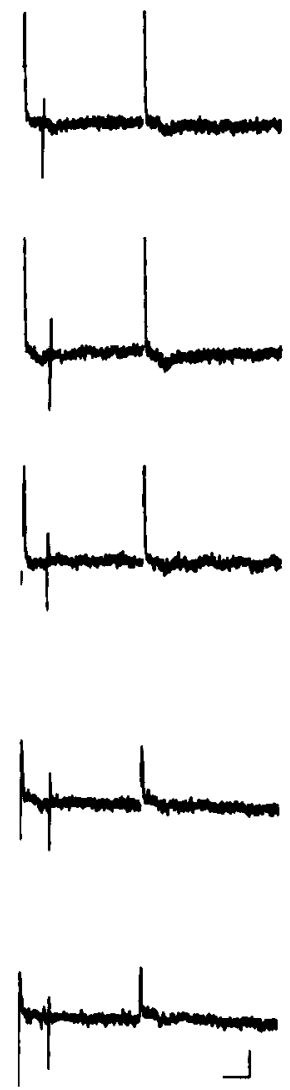

Figure 6. Examples of Type I responses to paired impulse stimulation of the corticostriatal pathway at interstimulus intervals of $20 \mathrm{msec}(A)$ and $100 \mathrm{msec}(B$ and $C$ ). The intensity of stimulation in $A$ and $B$ was approximately equal to threshold; suprathreshold stimulation was uscd in $C$. Calibration bars: $A, 100 \mu \mathrm{V}, 5 \mathrm{msec} ; B$ and $C, 100 \mu \mathrm{V}, 20 \mathrm{msec}$. The high- and lowpass filtering cut-offs were $300 \mathrm{~Hz}$ and $5 \mathrm{kHz}$, respectively. degree of inhibition exhibited in response to short interstimulus intervals (Fig. $5 C$, left and right panels; but see the results of suprathreshold stimulation below). In addition, the responses of Type II waveforms to paired impulse stimulation exhibited an inhibition-facilitation-inhibition pattern (Fig. $5 B$, right panel). This was the inverse of the pattern obscrved for Type I waveforms, so that when the probability of Type I spike discharge approached 0.00 , the probability of a Type II response approached 1.00 .

Suprathreshold stimulation. The use of suprathreshold stimulation intensities altered slightly the paired impulse responses of both Type I and Type II waveforms, but they remained qualitatively different and inversely related throughout the majority of interstimulus intervals tested. For example, when the probability of an action potential discharge to the first impulse of each pair was $>0.80$, Type I neurons exhibited an initial facilitatory response such that the average peak probability approached 1.00 in response to interstimulus intervals of $10-40$ msec (Fig. 8A, left panel), similar to that observed when lower stimulation intensities were used. Again similar to the effects observed with threshold intensities, the probability of Type I spike discharge was reduced to values approaching 0.00 in response to intervals of $60-250 \mathrm{msec}$ (Fig. 6C). However, at interstimulus intervals $>300 \mathrm{msec}$, the probability of spike firing approached and remained near 1.00 (Fig. 8A, right panel).

In response to high stimulation intensities, $73 \%$ of the Type II waveforms exhibited an initial inhibitory response (10-30 msec intervals), whereas this was seen in only $50 \%$ of the cases examined using threshold intensities (Fig. $8 B$, left panel). The magnitude of inhibition in response to these shorter intervals also was increased such that the probability of eliciting an action potential discharge was $0.03 \pm 0.02$ (Fig. $7 B$ ). Similar to the responses produced using near-threshold stimulation intensities, facilitation of Type II spike discharges first was seen in response to interstimulus intervals of approximately $40 \mathrm{msec}$ for all waveforms recorded. However, unlike the response patterns generated at threshold stimulation intensities, this facilitation never diminished, and the probability of Type II firing remained near 1.00 for intervals extending to $1000 \mathrm{msec}$ (Fig. $8 B$, right panel).

These characteristics of paired impulse responsiveness of Type I and Type II spike waveforms were remarkably stable across a number of different recording conditions. For example, it was noted above that the initial positivity of the Type II waveform, and thus its distinctiveness from the Type I class, can become degraded with changes in high-pass filtering, electrode impedance, or spike amplitude. Despite changes in such variables, however, Type I and Type II waveforms always display the paired impulses responses described above (see Fig. $9 A-D$, Type I; and $9 E-H$, Type II). As a result, during the course of these experiments we found that the paired impulse response profiles were better predictors of the Type I and Type II spike classcs than waveform. For example, when a cell first was being approached, spike amplitude was sometimes sufficiently small that there was ambiguity about cell classification based solely on waveform (Fig. 10). Results of paired impulse stimulation, however, always accurately predicted the action potential class after proximity of the electrode tip to the cell was increased to allow for unambiguous identification of the spike shape (Fig. 11).

We explicitly tested the possibility of utilizing the paired im- 

sponses to paired impulse stimulation of the corticostriatal pathway at interstimulus intervals of $20 \mathrm{msec}(A$ and $B$ ) and $100 \mathrm{msec}(C)$. The intensity of stimulation in $A$ and $C$ was approximately equal to threshold; suprathreshold stimulation was used in $B$. Calibration bars: $A$ and $B, 100 \mu \mathrm{V}, 5 \mathrm{msec}$; $C, 100 \mu \mathrm{V}, 20 \mathrm{msec}$. The high- and lowpass filtering cut-offs were $300 \mathrm{~Hz}$ and $5 \mathrm{kHz}$; respectivcly.
A
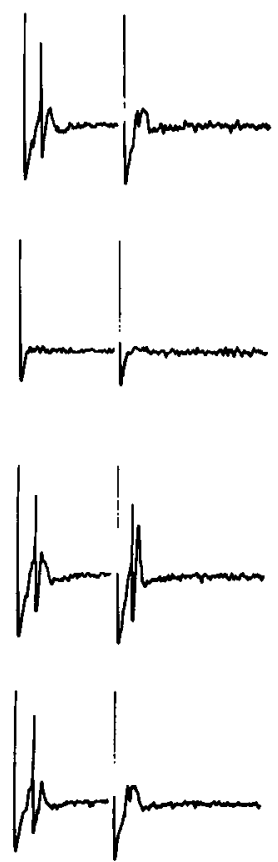

B
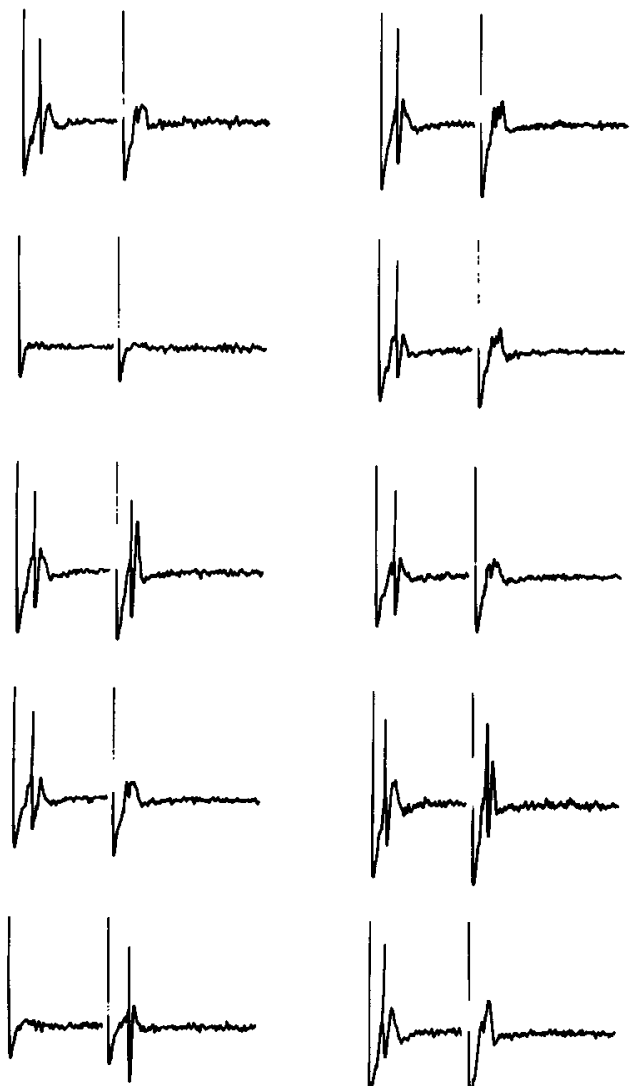

C
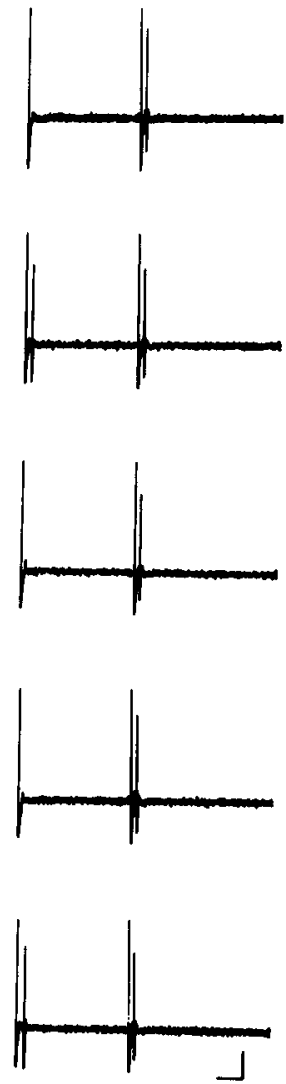

pulse profile to categorize striatal cell types independent of waveform with respect to the effects of electrode impedance. As noted earlier, when low-impedance electrodes were used, the initial positivity of Type II waveforms was greatly diminished, and very few cclls with initial positivitics werc detected. To determine whether this apparent change in the frequency of Type II waveforms represented an effect of electrode impedance on waveform (i.e., Type II waveforms still were being detected and isolated but could not be identified due to waveform distortion) or a selectivity of low-impedance electrodes (i.e., only Type I waveforms were being detected and isolated), we used 2 different ranges of electrode impedances (low, 1-2 M 2 ; high, 3.5-5.5 M $\Omega$ ) and determined the frequency with which cells were encountered that exhibited the Type I and Type II paired impulse functions. Electrodes were lowered through the striatum at 4 stereotaxically determined locations (see Materials and Methods). All waveforms $(n=137)$ were again localized on the basis of orthodromic activation via the corticostriatal pathway and were tested using paired impulse stimulation. Results showed that when a low-impedance electrode was used, an average of 0.75 Type I and 16.0 Type II paired impulse profiles was recorded per track; when a high-impedance electrode was used, 0.0 Type I and 17.5 Type II paired impulse profiles were recorded per track. Although in this particular test, no Type I paired impulse profiles were observed among the 70 cells encountered, it is possible to locate neurons exhibiting such profiles using high-impedance electrodes; a larger sample simply is needed. Thus, approximately the same ratios of the 2 paired impulse profiles were exhibited by waveforms detected with low and high impedances. The results of this analysis demonstrate 2 important points: (1) Paired impulse identification of striatal neurons can be made independent of spike waveform, and (2) striatal cells exhibiting the Type II paired impulse profile are detected much more frequently than cells exhibiting the Type I profile.

\section{Sensitivity of Type I and Type II waveforms to DA}

The waveform differences that characterize the Type I and Type II spike events also were observed consistently with 5-barrel micropipettes; thus, they are also independent of at least this difference in recording electrode type. In addition, although changes in the amplitudes of spikes were sometimes observed during iontophoretic application of agents, the fundamental characteristics that distinguish Type I and Type II neurons were not altered during the course of these experiments. Thus, the shapes of the waveforms also do not change as a function of firing rate either, at least with respect to the range of firing rates examined.

Iontophoretic application of DA inhibited Type I neurons when ejection currents of 40-70 nA were used to apply DA continuously in increasing amounts (Fig. 12, filled circles; Fig. $13, A, B)$. Type I neurons were never inhibited entirely by DA within the range of ejection currents tested, even when they were discharging at relatively low baseline rates. At lower ejection currents (5-30 nA), glutamate-evoked activity of Type I neurons either was unaffected by DA or displayed a slight excitation (Fig. 13A). Noncontinuous applications of DA resulted in the same effects as those observed when continuous appli- 


\section{A. TYPE I}

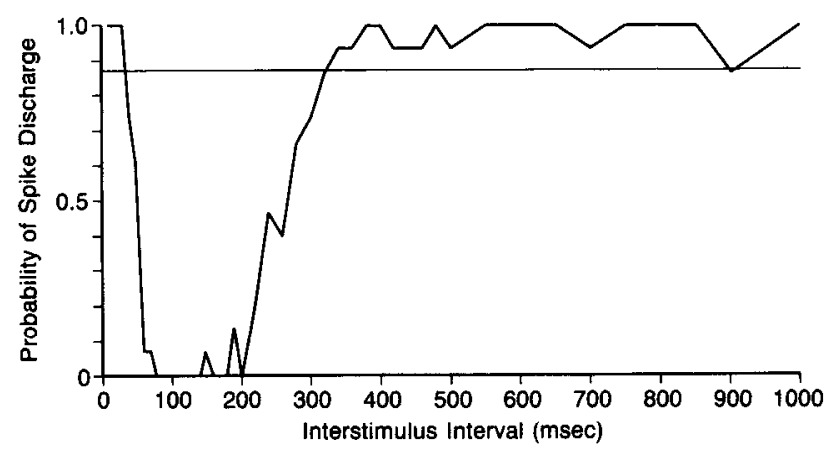

B. TYPE II

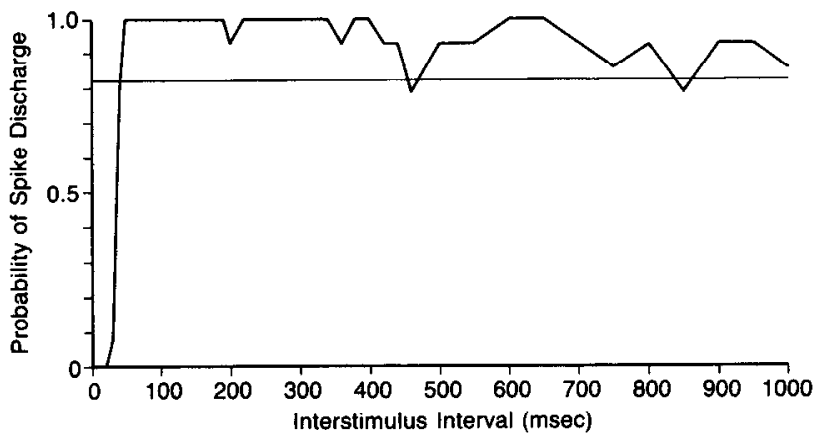

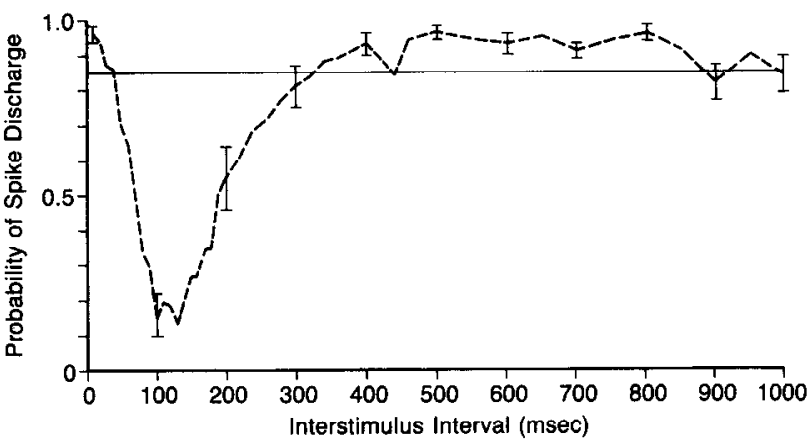

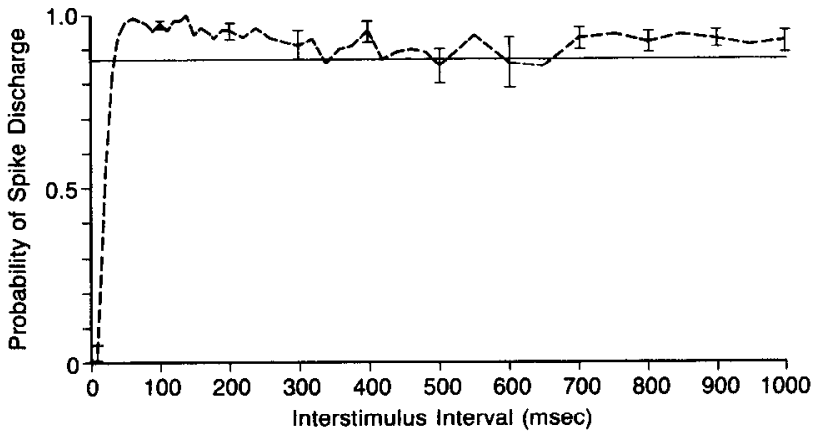

Figure 8. Paired impulse curves for striatal Type $\mathrm{I}(A)$ and Type II $(B)$ waveforms at suprathreshold stimulation intensities. The probabilites of spike discharge to the second impulse of each pair are presented in $A$ and $B$ as a function of interstimulus interval. Horizontal lines are equal to the probability of action potential discharge to the first impulse of all pairs of impulses. Left and right panels represent, respectively, single and average paired impulse profiles for Type I $(A)$ and Type II $(B)$ waveforms. The bars in the average paired impulse curves (right panels) indicate SEM.

cation was used (Fig. 12, open circles). Co-iontophoretic application of trifluoperazine (TFP; 5-10 nA) blocked the inhibitory effects of DA (40-60 nA) on all neurons tested.

Type II neurons exhibited approximately 5 times greater sensitivity to thc inhibitory effects of continuously applied DA than Type I neurons (Fig. 12, filled triangles). For example, unlike Type I neurons, glutamate-evoked activity of Type II cells was often inhibited completely by 10-20 nA of DA, regardless of the baseline firing rate (Fig. 13, C, D). In addition, ejection currents of $5 \mathrm{nA}$ significantly inhibited every Type II neuron tested, whereas the same ejection current never affected the firing of Type I cells. The differential sensitivity of Type I and Type II neurons to DA was confirmed by comparing the ejection currents necessary to produce $>50 \%$ inhibition for each cell type (Type I cells, $51 \pm 11 \mathrm{nA}$; Type II cells, $8 \pm 3 \mathrm{nA} ; t=$ $11.7, p<0.002)$. Greater sensitivity also was observed for Type II neurons tested with noncontinuous application of DA (Fig. 12 , open triangles). Type II neurons tested using both continuous and noncontinuous methods showed identical sensitivities. TFP (5-10 nA) also blocked the inhibitory effects of DA (10-15 nA) on Type II neurons.

From 5 separate preparations, 7 pairs of Type I and Type II neurons were recorded with the same electrode. The current necessary to produce $>50 \%$ inhibition was $50 \pm 10 \mathrm{nA}$ for Type I cells but only $8 \pm 3 \mathrm{nA}$ for Type II cells $(t=9.0, p<0.002)$. Thus, the differential sensitivity of the 2 cell types to DA cannot be attributed to different electrode characteristics.

These differences between Typc I and Type II neurons in sensitivity to DA were observed for action potentials of different amplitudes and correlated with spike waveform only. Moreover, the sensitivity differences did not depend on the distance of the recording electrode from the cell in cases when cells "drifted" toward or away from the electrode or when the electrode was moved to correct for such drift during the course of an experiment.

\section{Discussion}

\section{Functional identity of Type I and Type II striatal neurons}

The present study examined striatal Type I and Type II action potential waveforms with respect to their dependence on recording conditions, firing rate, response to paired impulse stimulation of the corticostriatal pathway, and sensitivity to DA. Results showed that the waveform shapes distinguishing these 2 action potential events are relatively independent of any distortions produced by the degree of capacitive coupling used during recording, the distance of the electrode tip from the target neuron, the type of electrode used (single versus multiple barrel), and the firing rate of the cell. The only variable that appears to be critical is electrode impedance; the marked positivity of the Type II waveform was greatly diminished when low-impedance electrodes were used.

These findings suggest that the shapes of Type I and Type II waveforms are not simply the products of differences in recording conditions or cell activity, and as such, it seems reasonable to ask whether the cells giving rise to the 2 waveforms also exhibit similar or different functional properties. The results of paired impulse stimulation of the corticostriatal pathway ad- 
A
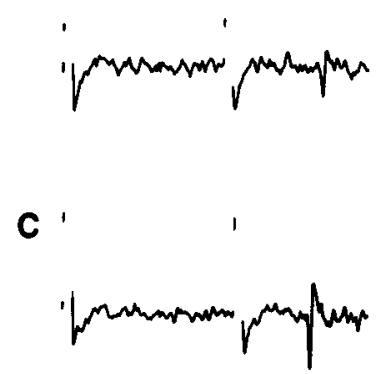

E

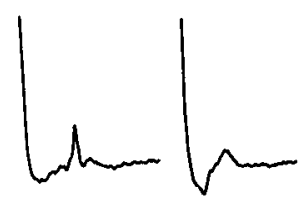

G

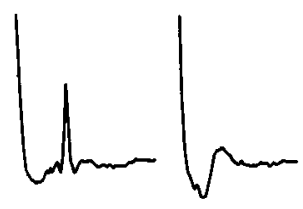

B

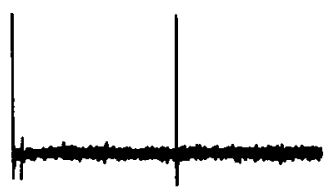

D

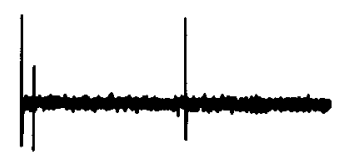

$\mathbf{F}$

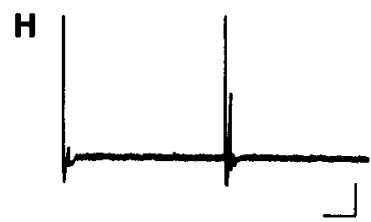

Figure 9. Examples of the effects of recording distance on Type I (A$D)$ and Type II $(E-H)$ waveform responses to paired impulse stimulation of the corticostriatal pathway at interstimulus intervals of $10 \mathrm{msec}(A$, $C, E$, and $G)$ and $100 \mathrm{msec}(B, D, F$, and $H)$. Note that regardless of the relative recording distance (i.e., amplitude of the waveform), the paired impulse responses of both Type I and Type II waveforms remain constant. Type I and Type II waveforms in this figure correspond to those in Figure 3. Calibration bars: $A-D, 50 \mu \mathrm{V} ; E-H, 100 \mu \mathrm{V} ; A, C$, $E$, and $G, 2 \mathrm{msec}, B, D, F$, and $H, 20 \mathrm{msec}$. The high- and low-pass filtering cut-offs were $300 \mathrm{~Hz}$ and $5 \mathrm{kHz}$, respectively.

dress this issue and demonstrate that Type I and Type II spikes represent action potential events with different functional characteristics. That is, the 2 waveforms exhibit qualitatively different paired impulse profiles to almost the entire range of interstimulus intervals tested. When stimulation intensities approximately equal to threshold were used, Type I waveforms showed a facilitation-inhibition-facilitation response profile (Fig. $5 A$, right panel). In marked contrast, the paired impulse curves of Type II waveforms were characterized by an inhibition-facilitation-inhibition pattern, which was the inverse of that found for Type I waveforms (Fig. $5 \mathrm{~B}$, right panel). As such, interstimulus intervals that increase the probability of a Type II spike discharge always decrease the probability of a Type I action potential. Similarly contrasting response pattcrns for Typc I and Type II waveforms are observed when greater stimulation intensities are used (Fig. 8, $A, B$, right panels). Together, these results demonstrate that the inverse polarities of Type $I$ and Type II waveforms observed using extracellular recording techniques cannot be attributed to differences in the orientation of the recording electrode to the same action potential generator, i.e., the 2 waveforms do not represent an active current sink and a passive source for the same action potential event. Instead,

Type I and Type II waveforms must arise from 2 action potential generators with differing response characteristics. This conclusion is further strenghthened by the difference found in latency to spike discharge in response to single impulse stimulation of cortical afferents. Type I waveforms are excited with a latency that is, on the average, 1-2 msec longer than Type II waveforms. Such a discrepancy is incompatible with an explanation of the 2 spike waveforms arising from the same action potential discharge.

It also is not likely that Type I and Type II waveforms represent a difference between somatodendritic spikes of intrinsic striatal neurons and axonal spikes of cortical fibers passing through the striatum but destined for nonstriatal targets. The Type II waveform is the only waveform which, due to its initial positivity, resembles an axonal spike. However, Type II spike generation must include a somatic contribution because Type II waveforms consistently include IS/SD breaks (Liles, 1974; Orr et al., 1987). Thus, the 2 waveforms must be generated from different cellular sources intrinsic to the striatum. This information, along with the present evidence for differential responses to paired impulse stimulation of cortical afferents, indicates that Type I and Type II waveforms must represent 2 functionally different subpopulations of striatal neurons.

Differences in the waveshapes of Type I and Type II neurons provide further information concerning the possible sites of generation for their action potentials. Both the initial negativity of Type I waveforms and the IS/SD components of Type II waveforms suggest that these waveforms represent recordings from striatal cell somas. However, although the initial negative-going phase of Type I waveforms indicates that their site of generation is proximal to the soma, the positive-going IS/SD components of Type II waveforms suggest that these spikes must be generated distal to the recording site at the soma. Similar conclusions have been drawn by Grace and Bunney (1983) with respect to the characteristic positive-going extracellular waveform of DA neurons within the substantia nigra.

\section{Paired impulse response of Type I and Type II neurons}

Previous studies have examined the effects of paired impulse stimulation of cortical afferents on the synaptic potentials generated in striatal neurons, but the results with regard to the presence of paired impulse inhibition were conflicting. Hull et al. (1973) and Vandermaelen and Kitai (1980) reported that relative EPSP amplitudes of striatal cells never were inhibited in response to paired impulse stimulation of cortical input and, in fact, at some interstimulus intervals (50-210 msec) were enhanced. In contrast, Kocsis and Kitai (1977) found that EPSP amplitudes were inhibited in response to paired impulse stimulation of cortex. Unlike any of these studies, Liles (1974), using extracellular recording techniques, found striatal neurons that exhibited facilitation to paired impulse stimulation at interstimulus intervals of $10-30 \mathrm{msec}$ and inhibition at intervals of 50$250 \mathrm{msec}$. This response profile is equivalent to that described in the present study for Type I neurons.

Resolving differences in results of the other studies is difficult because in only one of them (Hull et al., 1973) was the entire range of interstimulus intervals reported. Vandermaelen and Kitai (1980) suggested that these inconsistencies might be attributable to differences in anesthesia and surgical preparation. However, results from the present study suggest that either differences in the range of interstimulus intervals tested or differences in the intensity of stimulation could account for a failure 


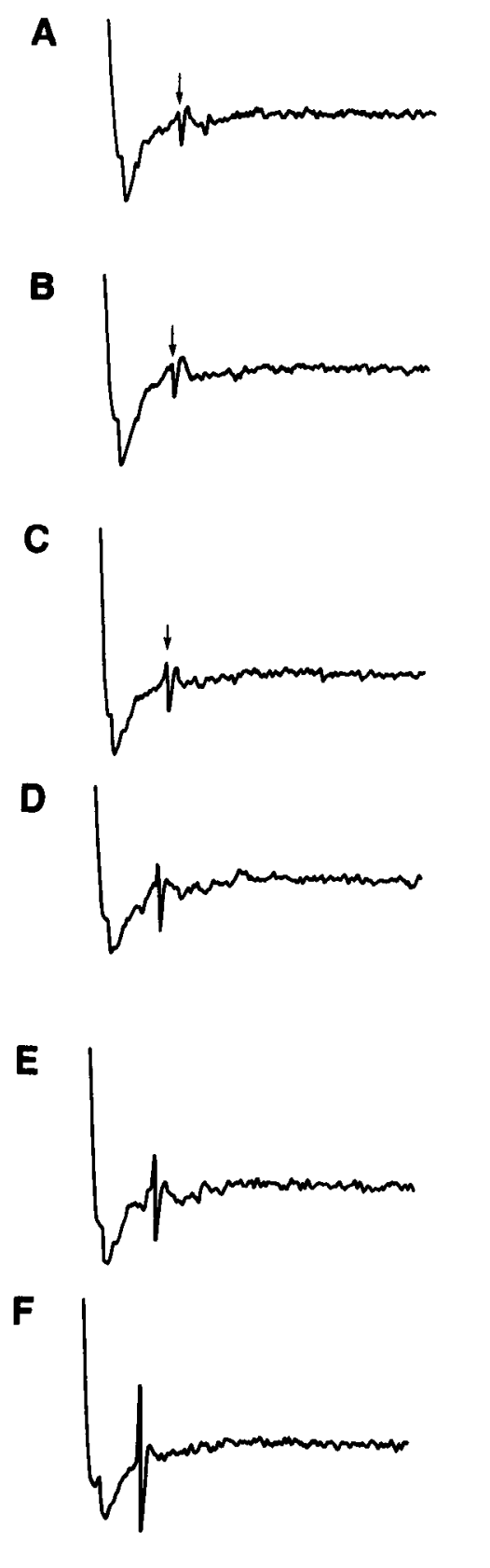

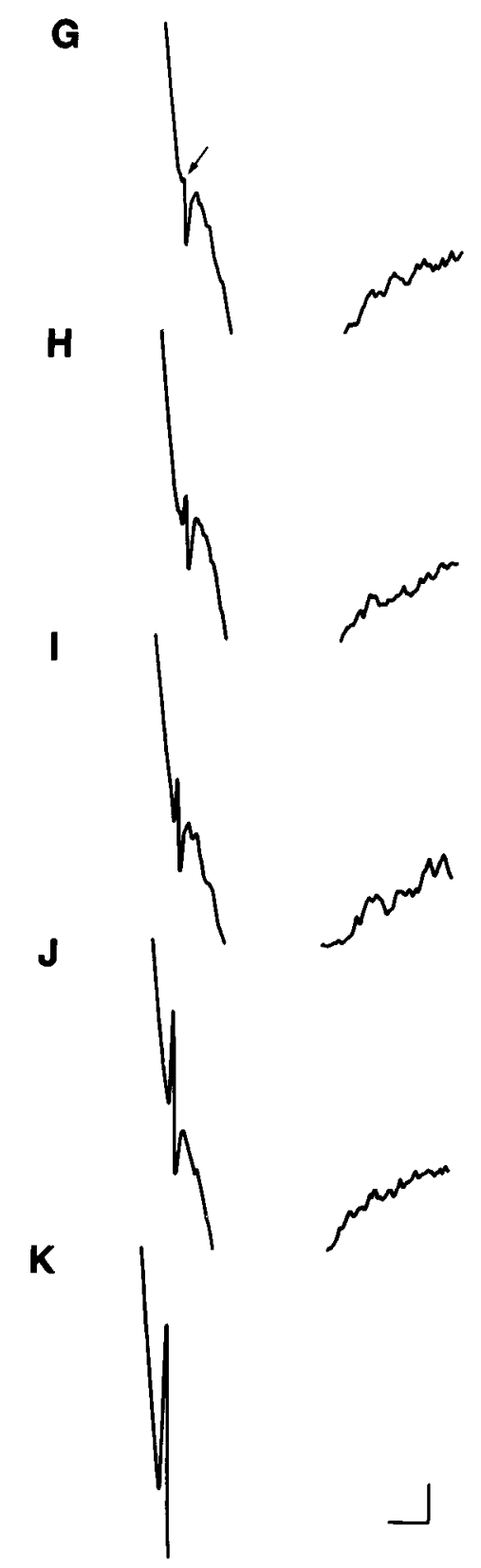

Figure 10. Examples of the effects of recording distance on the initial positivity associated with Type II waveforms. Arrows denote the degraded initial positivity when recording distances are relatively large (i.e., when spike amplitude is small and the potential ambiguity in waveform classification. Note that in this case, as the electrode is advanced toward the source of the action potential, an increase in the amplitude of the initial positivity is observed. $A-$ $F$ and $G-K$ are recordings from the same cell with the high-pass filtering cut-off set at $1 \mathrm{kHz}$ and $300 \mathrm{~Hz}$, respectively. Low-pass filtering was held constant at $5 \mathrm{kHz}$. Calibration bars, $50 \mu \mathrm{V}, 2 \mathrm{msec}$. to observe inhibitory responses to paired impulse stimulation. That is, inhibition of striatal cells (expressed as a decrease in EPSP amplitude) would not have been revealed in the above studies unless relatively short interstimulus intervals were tested. Type II neurons are inhibited most potently in response to interstimulus intervals of $10-30 \mathrm{msec}$, which is similar to the range of intervals (3-38 $\mathrm{msec}$ ) resulting in paired impulse inhibition of EPSP amplitude and probability of spike discharge in striatal cells recorded in vitro (Lighthall and Kitai, 1983). In addition, we found that the percentage of cells displaying inhibition nearly doubled when stimulation intensity was increased from threshold to suprathreshold.

With the exception of Liles' (1974) study, we feel it is unlikely that some of the previously reported negative findings with regard to paired impulse inhibition are a result of recordings from Type I neurons, which do not exhibit inhibition to short interstimulus intervals. This judgment is based on the fact that the same studies that failed to find paired impulse inhibition also reported facilitation of striatal cells to longer intervals of 50$210 \mathrm{msec}$ (Hull et al., 1973). Our results show that only Type II neurons exhibit facilitation to this same range of intervals, whereas Type I neurons display pronounced inhibition, and that some Type II cells can exhibit such facilitation in the absence of inhibition when threshold stimulation is used. Thus, differences in stimulation parameters, rather than differences in the cell type recorded, are likely to be the basis for discrcpancies in past reports.

It is notable that only one other study (Liles, 1974) besides the present one reported paired impulse responses of striatal neurons equivalent to those found for Type I neurons, and that study also used extracellular recording techniques. Results from the present experiments suggest that striatal cells exhibiting the Type II waveform paired impulse profiles are more numerous than those exhibiting the Type I waveform response curves, and 

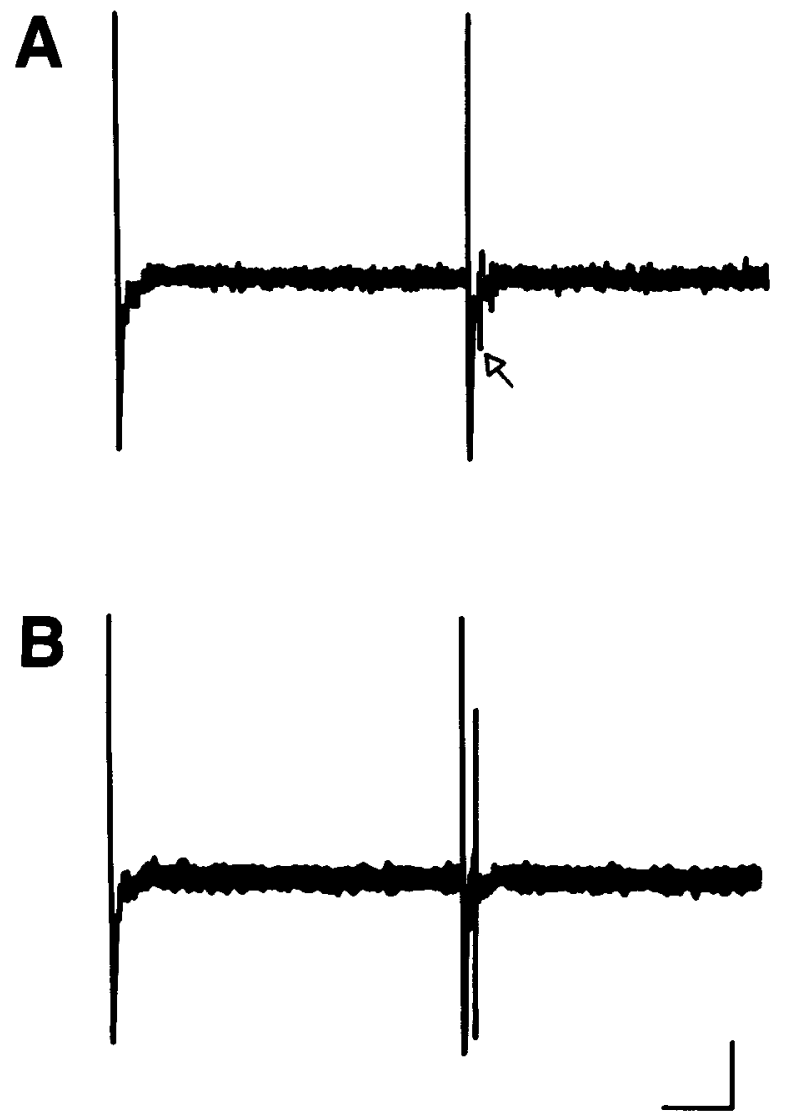

Figure 11. Examples of paired impulse responses of a Type II waveform that could not be classified initially. Arrow in $A$ denotes an action potential discharge that does not exhibit an initial positivity. However, the paired impulse response of this waveform at a $100 \mathrm{mscc}$ interstimulus interval indicates the spike belongs to the Type II class. As the electrode is advanced and spike amplitude increases $(B)$, the Type II waveshape is seen and the paired impulse response remains constant. The Type II waveform in this figure is the same as that in Figure 10. Calibration bars, $50 \mu \mathrm{V}, 20 \mathrm{msec}$. The high- and low-pass filtering cutoffs were $300 \mathrm{~Hz}$ and $5 \mathrm{kHz}$, respectively.

such a difference in proportions of the 2 cell classes may explain the relative paucity of Type I-like responses in prior investigations.

\section{Possible striatal connections underlying paired impulse responses of Type I and Type II neurons}

As outlined above, there is considerable evidence for recurrent inhibitory projections of striatal neurons. Anatomical and electrophysiological data suggest that these recurrent pathways involve axon collaterals from medium spiny cells that terminate on neighboring neurons (Park et al., 1980; Preston et al., 1980; Wilson and Groves, 1980; Fisher et al., 1986). The conduction time of such a pathway will be short, and thus its functional cffect will be reflected primarily in responses to short interstimulus intervals. The present paired impulse data show that such recurrent inhibition exists only for a subset of striatal neurons, specifically, those that exhibit a Type II extracellular waveform. In contrast, striatal neurons exhibiting the Type I waveform did not show inhibition to short interstimulus intervals even at suprathreshold intensities. Thus, the recurrent inhibitory collateral system of striatum is contained entirely within one of its subpopulations.

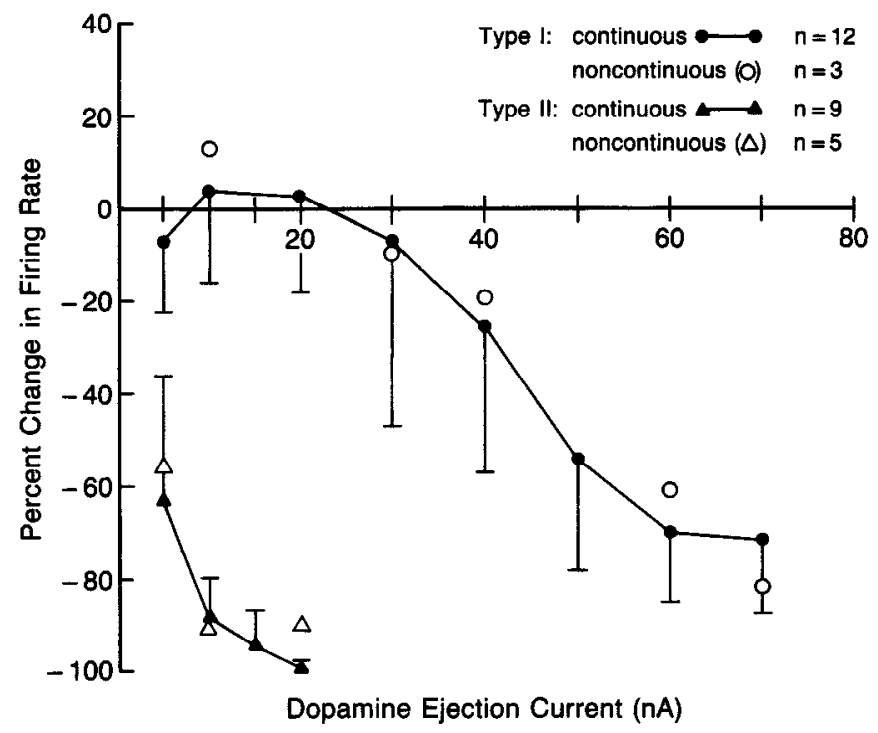

Figure 12. Dose-response relationship of striatal neurons to iontophoretic application of dopamine: Type I versus Type II. Effect of continuous application of dopamine is shown with solid symbols; effect of noncontinuous application of dopamine is shown with open symbols. All doses were applied for $3.5 \mathrm{~min}$. The first $30 \mathrm{sec}$ of each dose was not included in the calculation of percentage inhibition. Bars represent SD. Numbers in key represent the number of cells for each group.

These data also suggest that the Type II waveform is generated by the medium spiny striatal neuron, because it is this neuronal class that has been associated with the recurrent collateral network (Park et al., 1980; Preston et al., 1980; Wilson and Groves, 1980; Fisher et al., 1986). Furthermore, previous reports of enhanced responsiveness of medium spiny cells to longer interstimulus intervals (Hull et al., 1973; Vandermaelen and Kitai, 1980; see above) are also consistent with the facilitative responses of Type II waveforms observed at similar intervals in the present study (Figs. $5 B, 8 B$ ), providing further support for this hypothesis.

Whether or not Type I neurons also are medium spiny cells cannot be addressed by the present data. However, it is worth speculating that the Type I waveform reflects activity generated by a different striatal cell type. Approximately $95 \%$ of striatal neurons are of the medium spiny morphology, and several classes of aspiny neurons have been identified as among the remaining 5\% (Pasik et al., 1979; Chang et al., 1982). Although the bias in selectivity of recording electrodes precludes conclusions concerning proportions of different cell types, the high incidence of the Type II waveform and the low incidence of the Type I waveform are at least consistent with the hypothesis that the Type I waveform is generated by a non-medium spiny neuron.

The fact that the paired impulse curves for Type I and Type II neurons are inversely related is suggestive of an inhibitory synaptic relation between the 2 cell classes. Because Type II neurons respond to cortical stimulation with a latency that is shorter than that for Type I cells, it is possible that an inhibitory input from Type II onto Type I cells is the basis for this inverse relation. This hypothesis also requires that any such collaterals onto Type II neurons be shorter than the collaterals onto Type $I$ cells. Thus, at short interstimulus intervals $(10-30 \mathrm{msec})$, the inhibition exerted onto Type II cells would allow for a disinhibition of Type I ncurons; likewisc, at longer intervals (50-250 $\mathrm{msec}$ ), when Type II cells are facilitated, their inhibitory influ- 
A
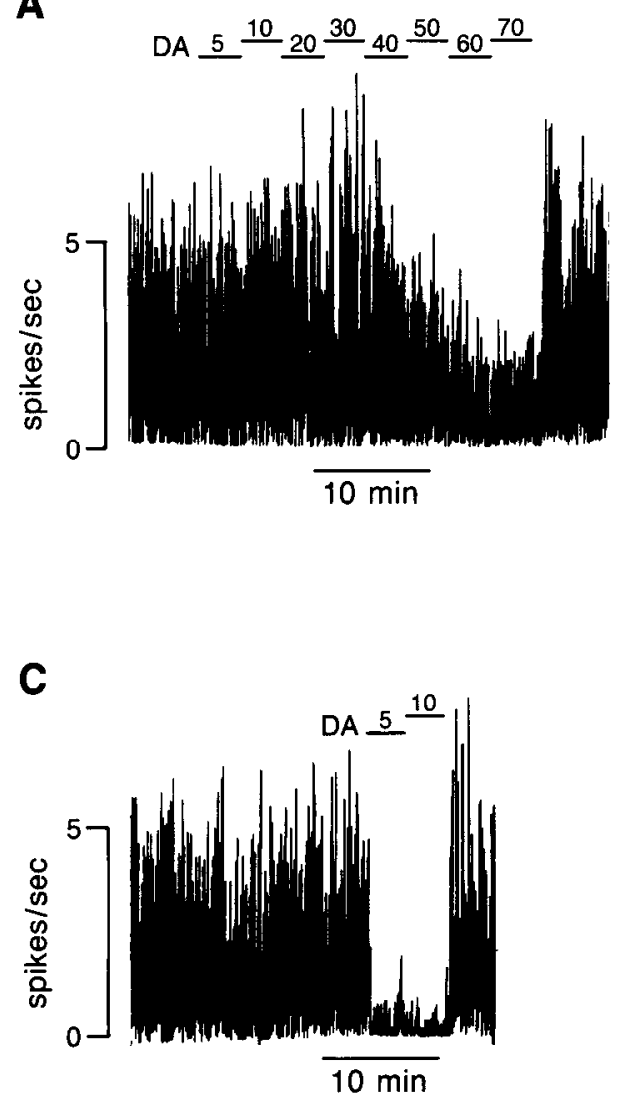

B
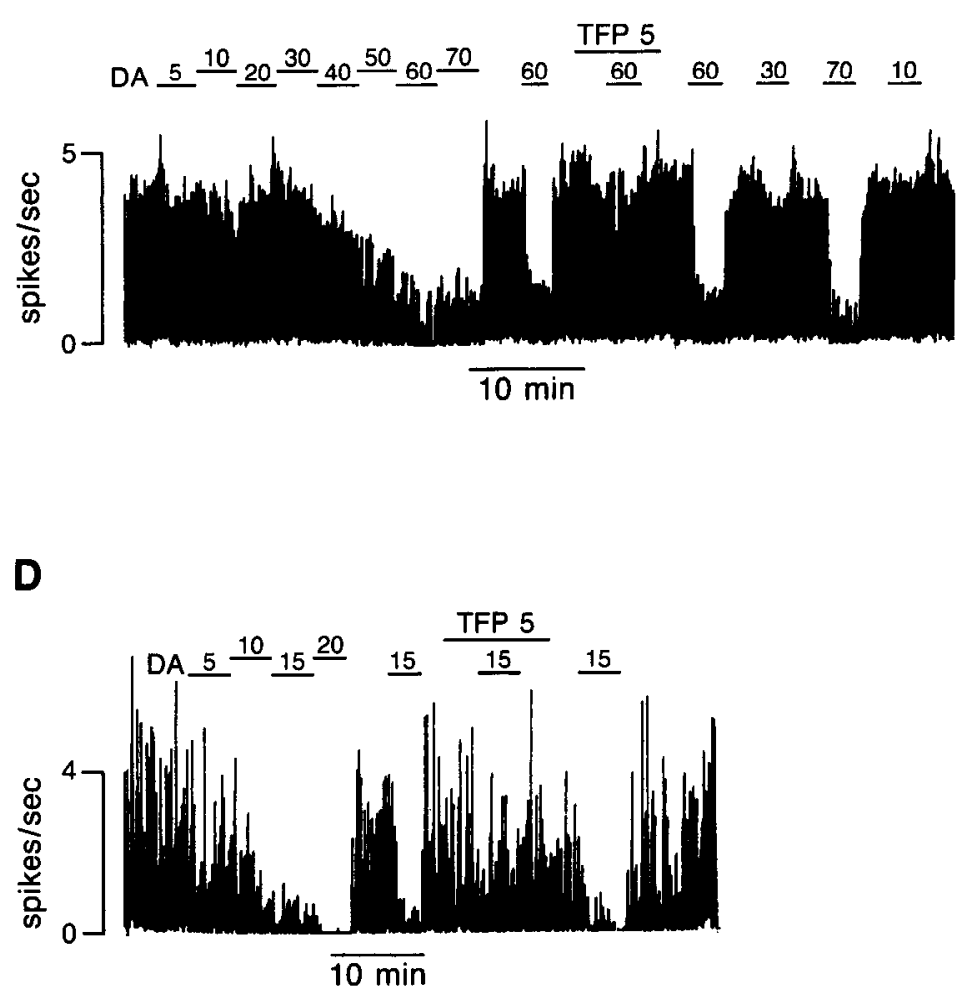

Figure 13. Chart records showing the effects of iontophoretically applied DA on the glutamate-induced discharge of 2 Type I $(A$ and $B)$ and 2 Type II waveforms $(C$ and $D$ ). Note that the firing of neither Type I waveform was inhibited by iontophoretic currents of 5-30 $\mathrm{nA}$ and that a slight excitation in firing rate occurred in response to this range of DA ejection currents for the cell shown in $A$. Neither cell was inhibited completely by DA delivered with a $70 \mathrm{nA}$ ejection current. Trifluoperizine (TFP) delivered simultaneously at $5 \mathrm{nA}$ blocked the inhibitory effects of DA for the cell shown in $B$. In contrast to the results shown for Type I waveforms, iontophoretic application of DA with ejection currents of 5-10 nA almost completely inhibited the firing of both Type II waveforms. For the cell shown in $D$, TFP applied simultaneously with DA, blocked its inhibitory effects.

ence on Type I neurons would be revealed. Although admittedly speculative, the dual characteristics of a short and monosynaptic connection could provide for negatively correlated spike discharge over the entire range of interstimulus intervals.

\section{Responses of Type I and Type II waveforms to DA}

Results of the iontophoretic studies showed that Type II neurons are much more sensitive to the inhibitory effects of DA than Type I neurons, but that DA also can be excitatory for the Type I cell class. These findings are consistent with the results of Johnson et al. (1983), who found that striatal cells responding with long latencies (>13 msec) to stimulation of the corticostriatal pathway were more likely to exhibit excitatory responses to iontophoretic application of DA than neurons responding with shorter latencies. Our results show that only Type I cells exhibit latencies of that magnitude, and only neurons of that class are excited by DA. These results also are consistent with our earlier observations that DA can enhance the excitatory effects of glutamate on Type I neurons (Chiodo and Berger, 1986).

Conclusive interpretation of the iontophoretic results is complicated by the inability to control the neuronal site of DA application. Although we did not see any dependence of excitation or inhibition on distance of the iontophoretic electrode from the recorded neuron, the possibility must be considered that the difference in sensitivity of Type I and Type II neurons is due in part to differences in the distance of the electrode from each cell type and, thus, the region of DA receptors (Herrling and Hull, 1980). However, the potential difference in sensitivity of the 2 cell classes to DA is supported by at least 2 other observations. First, the degree of dopaminergic denervation needed before the spontaneous activity of Type II neurons increases above control levels is significantly greater than the degree of denervation required for Type I cells (Orr et al., 1986, 1987). Second, recovery of motor movement after akinesiainducing DA depletions is associated with a return of Type II spontaneous activity to control levels (Nisenbaum et al., 1986), whereas Type I spontaneous activity remains elevated and does not correlate with behavioral recovery (Nisenbaum et al., in press). Moreover, both recovery at the behavioral and cellular levels is dependent on the residual DA-containing terminals because DA receptor antagonists given to recovered animals reinstate behavioral deficits and increased Type II cell activity to the same high levels observed just following the initial DAdepleting lesion. The greater sensitivity of Type II cells to DA could provide the basis for their decrease in spontaneous firing with behavioral recovery, as the cells respond to compensatory increases in the synthesis and release of DA from residual neu- 
rons that develop with time after the lesion (Zigmond and Stricker, 1985).

In summary, the present data argue for the existence of 2 functionally different subpopulations of striatal neurons with cortically driven activities that are reciprocally related. Although inconclusive, our data also are consistent with the notion that the proportion of the striatum comprised by each neuron class is markedly different: The Type II cell class may be much more numerous than the Type I cell class. Finally, several sources of evidence support the hypothesis that Type I and Type II neurons are differentially sensitive to DA, which could explain why they are affected differently by changes in dopaminergic input from the substantia nigra.

\section{References}

Bevan, P., C. M. Bradshaw, and E. Szabadi (1975) Effects of desipramine on neuronal responses to dopamine, noradrenaline, 5-hydroxytryptamine and acetylcholine in the caudate nucleus of the rat. $\mathrm{Br}$. J. Pharmacol. 54: 285-293.

Bloom, F. E., E. Costa, and G. C. Salmoiraghi (1965) Anesthesia and the responsiveness of individual neurones of the caudate nucleus of the cat to acetylcholine, norepinephrine and dopamine administered by microelectrophoresis. J. Pharmacol. Exp. Ther. 150: 244-252.

Bunney, B. S., and G. K. Aghajanian (1973) Electrophysiological effects of amphetamine on dopaminergic neurons. In Frontiers in Catecholamine Research, E. Usdin and S. Snyder, eds., pp. 957-962, Pergamon, New York.

Chang, H. T., C. J. Wilson, and S. T. Kitai (1982) A golgi study of rat neostriatal neurons: Light microscopic analysis. J. Comp. Neurol. 208: $107-126$.

Chiodo, L. A., and T. W. Berger (1986) Interactions between dopamine and amino acid-induced excitation and inhibition in the striatum. Brain Res. 375: 198-203.

Connor, J. D. (1970) Caudate nucleus neurones: Correlation of the effects of substantia nigra stimulation with iontophoretic dopamine. J. Physiol. (Lond.) 208: 691-703.

Feltz, P., and D. Albe-Fessard (1972) A study of an ascending nigrocaudate pathway. Electroencephalogr. Clin. Neurophysiol. 33: 179193.

Feltz, P., and J. DeChamplain (1972) Enhanced sensitivity of caudate neurones to microiontophoretic injections of dopamine in 6-hydroxydopamine treated cats. Brain Res. 43: 601-605.

Fisher, R. S., N. A. Buchwald, C. D. Hull, and M. S. Levine (1986) The GABAergic striatonigral neurons of the cat: Demonstration by double peroxidase labeling. Brain Res. 398: 148-156.

Gonzalez-Vegas, J. A. (1974) Antagonism of dopamine-mediated inhibition in the nigrostriatal pathway: A mode of action of some catatonia-inducing drugs. Brain Res. 80: 219-228.

Grace, A. A., and B. S. Bunney (1983) Intracellular and extracellular electrophysiology of nigral dopaminergic neurons-2. Action potential generating mechanisms and morphological correlates. Neuroscience 10: 317-331.

Graybiel, A. M., and C. W. Ragsdale Jr. (1983) Biochemical anatomy of the striatum. In Chemical Neuroanatomy, P. C. Emion, ed., pp. 427-504, Raven, New York.

Herrling, P. L., and C. D. Hull (1980) Iontophoretically applied dopamine depolarizes and hyperpolarizes the membrane of cat caudate neurones. Brain Res. 192: 441-462.

Hull, C. D., G. Bernardi, D. D. Price, and N. A. Buchwald (1973) Intracellular responses of caudate neurons to temporally and spatially combined stimuli. Exp. Neurol. 38: 324-336.
Johnson, S. W., M. R. Palmer, and R. Freedman (1983) Effects of dopamine on spontaneous and evoked activity of caudate neurons. Neuropharmacology 22: 843-851.

Kemp, J. M., and T. P. S. Powell (1971) The structure of the caudate nucleus of the cat: Light and electron microscopy. Phil. Trans. R. Soc. London 262: 383-401.

Kitai, S. T., A. Wagner, W. Precht, and T. Ohno (1975) Nigro-caudate and caudato-nigral relationship: An electrophysiological study. Brain Res. 85: 44-48.

Kitai, S. T., M. Sugimori, and J. D. Kocsis (1976) Excitatory nature of dopamine in the nigro-caudate pathway. Exp. Brain Res. 24: 351363.

Kocsis, J. D., and S. T. Kitai (1977) Dual excitatory inputs to caudate spiny neurons from substantia nigra stimulation. Brain Res. 138: 271283.

Lighthall, J. W., and S. T. Kitai (1983) A short duration GABAergic inhibition in identified neostriatal medium spiny neurons: In vitro slice study. Brain Res. Bull. 11: 103-110.

Liles, S. L. (1974) Single-unit responses of caudate neurons to stimulation of frontal cortex, substantia nigra, and entopeduncular nucleus in cats. J. Neurophysiol. 37: 254-265.

McLennan, H., and D. H. York (1967) The action of dopamine on neurones of the caudate nucleus. J. Physiol. (Lond.) 189: 393-402.

Nisenbaum, E. S., M. J. Zigmond, E. M. Stricker, and T. W. Berger (1986) Long-term effects of dopamine-depleting brain lesions on spontaneous activity of Type II striatal neurons: Relation to behavioral recovery. Brain Res. 398: 221-230.

Nisenbaum, E. S., M. J. Zigmond, E. M. Stricker, and T. W. Berger (in press) Spontaneous activity of Type II but not Type I striatal neurons is correlated with recovery of behavioral function after dopamine-depleting brain lesions. Brain Res.

Orr, W. B., T. W. Gardiner, E. M. Stricker, M. J. Zigmond, and T. W. Berger (1986) Short-term effects of dopamine-depleting brain lesions on spontaneous activity of Type II striatal neurons: Relation to local striatal dopamine levels and behavior. Brain Res. 376: 20-28.

Orr, W. B., E. M. Stricker, M. J. Zigmond, and T. W. Berger (1987) Short-term effects of dopamine-depleting brain lesions on spontaneous activity of Type I striatal neurons: Relation to local dopamine levels and behavior. Synapse 1: 461-469.

Park, M. R., J. W. Lighthall, and S. T. Kitai (1980) Recurrent inhibition in the rat neostriatum. Brain Res. 194: 359-369.

Pasik, P., T. Pasik, and M. Difigilia (1979) The internal organization of the neostriatum in mammals. In The Neostriatum, I. Divac and R. G. E. Oberg, eds., pp. 5-36, Pergamon, New York.

Preston, R. J., G. A. Bishop, and S. T. Kitai (1980) Medium spiny neuron projection from the rat striatum: An intracellular horseradish peroxidase study. Brain Res. 183: 253-263.

Skirboll, L. R., and B.S. Bunney (1979) The effects of acute and chronic haloperidol treatment on spontaneously firing neurons in caudate nucleus of the rat. Life Sci. 25: 1419-1434.

Vandermaelen, C. P., and S. T. Kitai (1980) Intracellular analysis of synaptic potentials in rat neostriatum following stimulation of the cerebral cortex, thalamus, and substantia nigra. Brain Res. Bull. 5: 725-733.

Wilson, C. J., and P. M. Groves (1980) Fine structure and synaptic connections of the common spiny neuron of the rat neostriatum: A study employing intracellular injection of horseradish peroxidase. $\mathrm{J}$. Comp. Neurol. 194: 599-615.

Wilson, C. J., and P. M. Groves (1981) Spontaneous firing patterns of identified spiny neurons in the rat neostriatum. Brain Res. 220: 67-80.

Zigmond, M. J., and E. M. Stricker (1985) Adaptive properties of monoaminergic neurons. In Handbook of Neurochemistry, Vol. 9, A. Lajtha, ed., pp. 87-102, Plenum, New York 